\title{
GEODESICS AND JACOBI FIELDS OF PSEUDO-FINSLER MANIFOLDS
}

\author{
MIGUEL ANGEL JAVALOYES AND BRUNO LEARTH SOARES
}

\begin{abstract}
In this paper, we derive the first and the second variation of the energy functional for a pseudo-Finsler metric using the family of affine connections associated to the Chern connection. This opens the possibility to accomplish computations with coordinate-free methods. Using the second variation formula, we introduce the index form and present some properties of Jacobi fields.
\end{abstract}

\section{INTRODUCTION}

Geodesics and Jacobi fields are probably the most important geometrical elements associated to a Finsler metric. Even though they can be defined without using any connection, choosing an appropriate connection associated to the Finsler metric can make it easier to get some properties of them. In particular, the main goal of this paper is to use the Chern connection to deduce some of these properties under the approach developed by H.-H. Matthias in [21, Definition 2.5], where the Chern connection is interpreted as a family of affine connections, namely, for every vector field $V$ in an open subset $\Omega \subset M$, non-zero everywhere, we get an affine connection $\nabla^{V}$. This affine connection is torsion-free and almost $g$-compatible, meaning that the derivative of the fundamental tensor is an expression in terms of the Cartan tensor (see subsection 2.3). Both properties allow one to compute the first and second variation of the energy functional in a coordinate-free manner. In this process, we will also use the further developments given in $[15,16]$, where a satisfactory relation between the curvature of the affine connection and the Chern curvature is obtained.

As one of our intentions is to promote the study of Finsler geometry between researchers of Riemannian background, we have included many details, with the purpose of providing in some cases index-free proofs or establishing the results in the very general setting of pseudo-Finsler metrics, apparently, the most general case where the Chern connection can be defined (see Remark 2.7). In particular the square of a Finsler metric is a pseudo-Finsler metric and the notions of indefinite Finsler metrics [5, 6] and Finsler spacetimes [26] can fit into this definition.

This work was partially supported by MINECO (Ministerio de Economía y Competitividad) and FEDER (Fondo Europeo de Desarrollo Regional) project MTM2012-34037, Regional J. Andalucía Grant P09-FQM-4496 and Fundación Séneca project 18942/JLI/13. This research has been supported by the programme "Young leaders in research" by Fundación Séneca, Regional Agency for Science and Technology from the Region of Murcia. This work has been finsihed during a visit of the first author to the University of São Paulo supported by the Fapesp grant for visiting researchers with process 2013/1070-7.

2000 Mathematics Subject Classification: Primary 53C22, 53C50, 53C60, 58B20

Key words: Finsler, geodesics, Chern connection, Jacobi fields. 
The fundamental objective of this paper is to provide a computation of the first and the second variation of the energy functional of a pseudo-Finsler metric (Propositions 3.1 and 3.2). As a first step, we prove that geodesics are the critical points of the energy functional when we consider curves with fixed endpoints, or, more generally, with endpoints in two submanifolds $P$ and $Q$ (Corollary 3.7). Moreover, the second variation formula allows us to define the index form and the Jacobi fields (subsection 3.7), and with our approach to the Chern connection we can straightforwardly deduce some basic properties of Jacobi fields (see subsection $3.4)$ and characterize the kernel of the index form as the $(P, Q)$-Jacobi fields along $\gamma$ (Proposition 3.11).

The paper is structured as follows. Section 2 contains some basic results about pseudo-Finsler metrics including some properties of its fundamental tensor, the Cartan tensor and the Chern connection. We also introduce several basic notions: parallelism of a vector field along a curve, geodesics, namely, curves having parallel tangent vector fields, and the exponential map. In the last subsection we recall some properties concerning the curvature of the Chern connection obtained in $[15,16]$, in particular its relation to the Chern curvature.

In Section 3 we compute the first and the second variation of the energy functional (Propositions 3.1 and 3.2) and then we get the index form when the boundary conditions are given by two submanifolds. As a preparatory step, in subsection 3.1 we collect some definitions and properties of submanifolds of pseudo-Finsler manifolds. Then, in subsection 3.4, we give some properties of Jacobi fields.

\section{Pseudo-Finsler metrics And the Chern Connection}

2.1. Preliminaries on pseudo-Finsler metrics. Let $M$ be an $n$-dimensional manifold and denote by $\pi: T M \rightarrow M$ the natural projection of the tangent bundle $T M$ into $M$. Let $A \subset T M \backslash \mathbf{0}$ be an open subset of $T M$ which is conic, that is, such that $\pi(A)=M$ and $\lambda v \in A$, for every $v \in A$ and $\lambda>0$. We say that a smooth function $L: A \rightarrow \mathbb{R}$ is a (conic, two homogeneous) pseudo-Finsler metric if

(i) $L$ is positive-homogeneous of degree 2, that is, $L(\lambda v)=\lambda^{2} L(v)$ for every $v \in A$ and $\lambda>0$,

(ii) for every $v \in A$, the fundamental tensor $g_{v}$ of $L$ at $v$ defined by

$$
g_{v}(u, w):=\left.\frac{1}{2} \frac{\partial^{2}}{\partial t \partial s} L(v+t u+s w)\right|_{t=s=0},
$$

for any $u, w \in T_{\pi(v)} M$, is nondegenerate.

Clearly, the fundamental tensor is bilinear and symmetric. We will refer to the pair $(M, L)$, being $M$ a manifold and $L$ a pseudo-Finsler metric on $M$, as a pseudoFinsler manifold.

Remark 2.1. In the following we shall omit the adjectives two-homogeneous and conic whenever there is no danger of misunderstanding. In [18] the same name of pseudo-Finsler metrics is used for a somewhat different concept. In the cited reference, a pseudo-Finsler metric is not allowed to be non-positive away from the zero section and it is positive homogeneous of degree one. Moreover, its fundamental tensor is not necessarily nondegenerate. Nevertheless, if $F: A_{1} \subset T M \rightarrow[0,+\infty)$ is a conic pseudo-Finsler metric on $M$ as in [18] and

$$
\tilde{A}_{1}=\left\{v \in A_{1} \backslash \mathbf{0}: \text { the fundamental tensor } g_{v} \text { of } F^{2} \text { is nondegenerate }\right\},
$$


then $L=\left.F^{2}\right|_{\tilde{A}_{1}}: \tilde{A}_{1} \rightarrow(0,+\infty)$ fits in our definition of pseudo-Finsler metric. Moreover, if $L: A_{2} \subset T M \backslash \mathbf{0} \rightarrow \mathbb{R}$ is a pseudo-Finsler metric on $M$ as defined above and $\tilde{A}_{2}=\left\{v \in A_{2}: L(v) \neq 0\right\}$, then $F=\left.\sqrt{|L|}\right|_{\tilde{A}_{2}}: \tilde{A}_{2} \rightarrow(0,+\infty)$ is a conic pseudo-Finsler metric on $M$ as in [18] (extending $F$ continuously to the zero section if necessary). Note finally that the concept of pseudo-Finsler metrics in [18] is particularly convenient when one is interested in studying distance properties.

We mention some particular cases of pseudo-Finsler metrics:

(i) if $A=T M \backslash \mathbf{0}$ and the fundamental tensor is positive definite, then $L$ is positive away from the zero section and $F=\sqrt{L}$ is what traditionally has been called a Finsler metric;

(ii) if $A \subsetneq T M \backslash \mathbf{0}$, but the fundamental tensor is positive definite, then the square root $F=\sqrt{L}$ is called a conic Finsler metric in [18];

(iii) if the fundamental tensor has index one, then $L$ is called a Lorentzian Finsler metric (see $[1,12,19])$. This is also the case of Finsler spacetimes where some authors ask $L$ to be defined in the whole $T M[5,6,26]$.

Remark 2.2. Even though sometimes the domain of definition can change (see Remark 2.1), from now on, by abuse of notation, we omit the subset $A$ when fixing a (conic) pseudo-Finsler manifold $(M, L)$, assuming that $L$ is defined in $A$.

We end this subsection with a proposition that describes the positive homogeneity of the fundamental tensor of a pseudo-Finsler metric as well as some relations between the fundamental tensor and the metric. It is a simple consequence of basic properties of homogeneous functions.

Proposition 2.3. Given a pseudo-Finsler metric $L$ and $v \in A$, the fundamental tensor $g_{v}$ is positive homogeneous of degree 0, that is, $g_{\lambda v}=g_{v}$ for $\lambda>0$. Moreover $g_{v}(v, v)=L(v)$ and $g_{v}(v, w)=\left.\frac{1}{2} \frac{\partial}{\partial z} L(v+z w)\right|_{z=0}$.

2.2. Cartan tensor. In Finsler geometry, unlike the Riemannian setting, we need to consider the third derivatives of the metric in order to define a connection. This information is contained in the Cartan tensor, which is defined as the trilinear form

$$
C_{v}\left(w_{1}, w_{2}, w_{3}\right)=\left.\frac{1}{4} \frac{\partial^{3}}{\partial s_{3} \partial s_{2} \partial s_{1}} L\left(v+\sum_{i=1}^{3} s_{i} w_{i}\right)\right|_{s_{1}=s_{2}=s_{3}=0},
$$

for $v \in A$ and $w_{1}, w_{2}, w_{3} \in T_{\pi(v)} M$. Observe that $C_{v}$ is symmetric, that is, its value does not depend on the order of $w_{1}, w_{2}$ and $w_{3}$.

Remark 2.4. Let $\pi_{A}: A \rightarrow M$ be the restriction to $A$ of the natural projection $\pi: T M \rightarrow M$. Now let $\pi_{A}^{*}\left(T^{*} M\right)$ be the fiber bundle over $A$ induced by the natural projection of the cotangent bundle $\pi^{*}: T^{*} M \rightarrow M$ through $\pi_{A}$. Observe that the fundamental tensor is a symmetric section of the fiber bundle $\pi_{A}^{*}\left(T^{*} M\right) \otimes$ $\pi_{A}^{*}\left(T^{*} M\right)$. Moreover, the Cartan tensor is a symmetric section of the fiber bundle $\pi_{A}^{*}\left(T^{*} M\right) \otimes \pi_{A}^{*}\left(T^{*} M\right) \otimes \pi_{A}^{*}\left(T^{*} M\right)$.

Furthermore, the Cartan tensor can be obtained from the fundamental tensor as

$$
C_{v}\left(w_{1}, w_{2}, w_{3}\right)=\left.\frac{1}{2} \frac{\partial}{\partial z} g_{v+z w_{1}}\left(w_{2}, w_{3}\right)\right|_{z=0} .
$$


If $g$ is an arbitrary symmetric section of $\pi_{A}^{*}\left(T^{*} M\right) \otimes \pi_{A}^{*}\left(T^{*} M\right)$ that is positive homogeneous of degree zero $\left(g_{v}=g_{\lambda v}\right.$ for $\left.\lambda>0\right)$ we define its Cartan tensor as above.

The following is another simple consequence of basic properties of homogeneous functions:

Proposition 2.5. The Cartan tensor is homogeneous of degree -1 , that is, $C_{\lambda v}=$ $\frac{1}{\lambda} C_{v}$ for any $v \in A$ and $\lambda>0$. Moreover, $C_{v}\left(v, w_{1}, w_{2}\right)=0$ for every $w_{1}, w_{2} \in$ $\stackrel{T}{\pi(v)} M$.

To conclude this subsection, we state a well-known result that appears, for example, in [3, Theorem 3.4.2.1].

Proposition 2.6. An arbitrary (non-degenerate) symmetric section $g$ of $\pi_{A}^{*}\left(T^{*} M\right) \otimes \pi_{A}^{*}\left(T^{*} M\right)$ that is positive homogeneous of degree zero comes from a pseudo-Finsler metric if and only if its Cartan tensor is symmetric.

Remark 2.7. An arbitrary symmetric section $g$ of $\pi_{A}^{*}\left(T^{*} M\right) \otimes \pi_{A}^{*}\left(T^{*} M\right)$ that is positive homogeneous of degree zero is usually known as a generalized metric. It was introduced by A. Moor in 1956 [24] and studied in detail by J. R. Vanstone [29], M. Hashiguchi [14], R. Miron [23] and others. For a quite recent survey, we refer to [20]. Unfortunately, the Chern connection is not well-defined for generalized metrics unless they come from a pseudo-Finsler metric. This is because the following remark is essential to prove existence of a connection which is torsion-free and almost metric compatible (see for example [15, Proposition 2.3]).

Remark 2.8. In the case of a pseudo-Finsler manifold $(M, L)$, the Cartan tensor is symmetric. This together with Proposition 2.5 means that $C_{v}\left(v, w_{1}, w_{2}\right)=$ $C_{v}\left(w_{1}, v, w_{2}\right)=C_{v}\left(w_{1}, w_{2}, v\right)=0$ for any $v \in A$ and $w_{1}, w_{2} \in T_{\pi(v)} M$.

2.3. Chern connection and covariant derivative. Assume that $(M, L)$ is a pseudo-Finsler manifold with domain $A \subset T M$, and denote by $\mathfrak{X}(\Omega)$ the module of smooth vector fields on an open subset $\Omega \subset M$. We say that $V \in \mathfrak{X}(\Omega)$ is $L$-admissible if $V(p) \in A$ for every $p \in \Omega$. Then the mapping

$$
\left\{\begin{array}{l}
g_{V}:(X, Y) \in \mathfrak{X}(\Omega) \times \mathfrak{X}(\Omega) \mapsto g_{V}(X, Y) \in C^{\infty}(\Omega), \\
g_{V}(X, Y)(p):=g_{V(p)}(X(p), Y(p)) \quad(p \in \Omega)
\end{array}\right.
$$

is a pseudo-Riemannian metric on $\Omega$. We construct in the same way a type $(0,3)$ tensor field $C_{V}$ on $\Omega$ from the Cartan tensor.

It can be associated to any $L$-admissible vector field $V \in \mathfrak{X}(\Omega)$ an affine connection

such that

$$
\nabla^{V}: \mathfrak{X}(\Omega) \times \mathfrak{X}(\Omega) \rightarrow \mathfrak{X}(\Omega)
$$

(i) $\nabla_{X}^{V} Y-\nabla_{Y}^{V} X=[X, Y]$ for all $X, Y \in \mathfrak{X}(\Omega)$ (torsion freeness);

(ii) $X\left(g_{V}(Y, Z)\right)=g_{V}\left(\nabla_{X}^{V} Y, Z\right)+g_{V}\left(Y, \nabla_{X}^{V} Z\right)+2 C_{V}\left(\nabla_{X}^{V} V, Y, Z\right)$ for all $X, Y, Z \in$ $\mathfrak{X}(\Omega)$ (almost g-compatibility).

We say that $\nabla^{V}$ is the Chern connection of $(M, L)$ associated to the $L$-admissible vector field $V \in \mathfrak{X}(\Omega)$. It is easy to see that $\nabla^{V}$ is positive homogeneous of degree 0 in $V$, i.e., $\nabla^{V}=\nabla^{\lambda V}$ for all positive $\lambda$.

Now we suppose that $\Omega$ is a chart domain with coordinate system

$$
x=\left(x^{1}, \ldots, x^{n}\right): \Omega \rightarrow x(\Omega) \subset \mathbb{R}^{n} .
$$


The Christoffel symbols of $\nabla^{V}$ with respect to the chart $(\Omega, x)$ are the smooth functions $\Gamma_{i j}^{k}(V): \Omega \rightarrow \mathbb{R}$ such that

$$
\nabla_{\frac{\partial}{\partial x^{i}}}^{V}\left(\frac{\partial}{\partial x^{j}}\right)=\sum \Gamma_{i j}^{k}(V) \frac{\partial}{\partial x^{k}} ; \quad i, j \in\{1, \ldots, n\} .
$$

In fact, $\Gamma_{i j}^{k}(V)=\Gamma_{i j}^{k} \circ V$ where $\Gamma_{i j}^{k}: \pi^{-1}(\Omega) \cap A \rightarrow \mathbb{R}$ are the Christoffel symbols of the Chern connection (see [15, Proposition 2.6]).

Given a smooth curve $\gamma:[a, b] \rightarrow M$, we denote by $\mathfrak{X}(\gamma)$ the $C^{\infty}([a, b])$-module of vector fields along $\gamma$. We say that $W \in \mathfrak{X}(\gamma)$ is $L$-admissible if $W(t) \in A$ for all $t \in[a, b]$. For every $L$-admissible vector field $W \in \mathfrak{X}(\gamma)$, the Chern connection induces a covariant derivative $D_{\gamma}^{W}: \mathfrak{X}(\gamma) \rightarrow \mathfrak{X}(\gamma)$ along $\gamma$, given locally, when $\gamma$ is contained in the chart domain $\Omega$, by

$$
D_{\gamma}^{W} X=\sum_{i=1}^{n}\left(\dot{X}^{k}+\sum_{i, j=1}^{n} X^{i} \dot{\gamma}^{j}\left(\Gamma_{i j}^{k} \circ W\right)\right)\left(\frac{\partial}{\partial x^{k}} \circ \gamma\right),
$$

where $X=\sum_{i=1}^{n} X^{i}\left(\frac{\partial}{\partial x^{i}} \circ \gamma\right), \dot{\gamma}=\sum_{i=1}^{n} \dot{\gamma}^{i}\left(\frac{\partial}{\partial x^{i}} \circ \gamma\right)$ (see, again, [15, Proposition 2.6]). The induced covariant derivative is also almost $g$-compatible in the sense that

$$
\left(g_{W}(X, Y)\right)^{\prime}=g_{W}\left(D_{\gamma}^{W} X, Y\right)+g_{W}\left(X, D_{\gamma}^{W} Y\right)+2 C_{W}\left(D_{\gamma}^{W} W, X, Y\right),
$$

for all $X, Y \in \mathfrak{X}(\gamma)$.

2.4. Parallel vector fields. Having been defined the induced covariant derivative, we can introduce the concept of parallelism along a (piecewise) smooth curve $\gamma$ : $[a, b] \rightarrow M$. Then, of course, we have to assume that $\gamma$ is also $L$-admissible in the sense that $\dot{\gamma}(t) \in A$ for all $t \in[a, b]$. (At break points, both velocity vectors of $\gamma$ must belong to $A$.) For simplicity, we may assume that $\operatorname{Im}(\gamma)$ is in the domain of a chart $(\Omega, x)$; this assumption is clearly not restrictive.

Definition 2.9. Let $(M, L)$ be a pseudo-Finsler manifold and $\gamma$ a smooth $L$ admissible curve in $M$. A vector field $X \in \mathfrak{X}(\gamma)$ is called parallel if $D_{\gamma}^{\dot{\gamma}} X=0$.

Proposition 2.10. Hypotheses and notation as above. Given a vector $w \in T_{\dot{\gamma}(a)} M$, there is a unique parallel vector field $X$ along $\gamma$ such that $X(a)=w$.

The proof is routine.

\subsection{Geodesics and the exponential map.}

Definition 2.11. A smooth $L$-admissible curve $\gamma$ of a pseudo-Finsler manifold $(M, L)$ is called a geodesic if its velocity vector field $\dot{\gamma}$ is parallel along $\gamma$.

In terms of local coordinates, the geodesic equation is of the form

$$
\ddot{\gamma}^{k}+\sum_{i, j=1}^{n} \dot{\gamma}^{i} \dot{\gamma}^{j}\left(\Gamma_{i j}^{k} \circ \dot{\gamma}\right)=0, \quad k \in\{1, \ldots, n\} .
$$

As in the pseudo-Riemannian case, we have:

Proposition 2.12. Let $(M, L)$ be a pseudo-Finsler manifold. For every $v \in A$, there exists a unique (maximal) geodesic $\gamma_{v}:[a, b) \rightarrow M$ such that $\dot{\gamma}_{v}(0)=v$, $b \in(0,+\infty]$. 
Remark 2.13. If $\gamma:[a, b] \rightarrow M$ is a geodesic of $(M, L)$, then the function $L \circ \dot{\gamma}$ : $[a, b] \rightarrow \mathbb{R}$ is constant. Indeed, taking into account the almost $g$-compatibility of the induced covariant derivative and Remark 2.9, we have $(L \circ \dot{\gamma})^{\prime}=2 g_{\dot{\gamma}}\left(D_{\gamma}^{\dot{\gamma}} \dot{\gamma}, \dot{\gamma}\right)=0$. If $L \circ \dot{\gamma}=0$, then $\gamma$ is called a lightlike geodesic.

Definition 2.14. Let $(M, L)$ be a pseudo-Finsler manifold. If $p \in M$, let $\mathcal{D}_{p}$ be the set of vectors $v$ in $A \cap T_{p} M$ satisfying the following condition: if $\gamma_{v}:[0, b) \rightarrow M$ is the maximal geodesic such that $\dot{\gamma}_{v}(0)=v$, then $b>1$. The exponential map of $(M, L)$ at $p$ is the mapping

$$
\exp _{p}: \mathcal{D}_{p} \rightarrow M, \quad v \mapsto \exp _{p}(v):=\gamma_{v}(1) .
$$

Proposition 2.15. The domain of the exponential map $\exp _{p}: \mathcal{D}_{p} \rightarrow M$ is an open subset of $A \cap T_{p} M$, and $\exp _{p}$ is smooth on $\mathcal{D}_{p}$. If $A \cap T_{p} M=T_{p} M \backslash\{0\}$, then $\exp _{p}$ is defined in an open subset of $0_{p}$, putting $\exp _{p}\left(0_{p}\right)=p$.

Proof. The first statement is a consequence of the theorem on smooth dependence on the initial data of ODEs. To see the second statement, observe that the functions

$$
v \in T_{p} M \backslash\{0\} \mapsto \sum v^{i} v^{j} \Gamma_{i j}^{k}(v) \in \mathbb{R} ; \quad k \in\{1, \ldots, n\}
$$

are positive-homogeneous of degree 2 , so they can be extended to $C^{1}$ functions at zero. Indeed, homogeneous functions of positive degree can be extended continuously to zero as zero, and the derivative of a homogeneous function of degree 2 is a homogeneous function of degree 1 (see also Proposition 2.3). In fact, the extensions will be of class $C^{1}$ on $\pi^{-1}(\Omega)$.

2.6. Jacobi operator and flag curvature. If we fix an $L$-admissible vector field $V$ in $\Omega \subset M$, the Chern connection $\nabla^{V}$ is an affine connection on $\Omega$, whose curvature tensor is given by

$$
R^{V}(X, Y) Z=\nabla_{X}^{V} \nabla_{Y}^{V} Z-\nabla_{Y}^{V} \nabla_{X}^{V} Z-\nabla_{[X, Y]}^{V} Z \quad ; \quad X, Y, Z \in \mathfrak{X}(\Omega) .
$$

The $\nabla^{V}$-covariant derivative of the Cartan tensor $C_{V}$ is the $(0,4)$ tensor defined by

$$
\begin{aligned}
\nabla_{X}^{V} C_{V}(Y, Z, W)=X\left(C_{V}(Y, Z, W)\right)- & C_{V}\left(\nabla_{X}^{V} Y, Z, W\right) \\
& -C_{V}\left(Y, \nabla_{X}^{V} Z, W\right)-C_{V}\left(Y, Z, \nabla_{X}^{V} W\right) .
\end{aligned}
$$

It is straightforward to check that $\nabla_{X}^{V} C_{V}$ is trilinear, symmetric and

$$
\nabla_{X}^{V} C_{V}(V, Z, W)=-C_{V}\left(\nabla_{X}^{V} V, Z, W\right) .
$$

Moreover, for every $X, Y, Z, W \in \mathfrak{X}(\Omega)$, the curvature tensor has the following symmetries (see [15, Proposition 3.1]):

(ii)

$$
R^{V}(X, Y)=-R^{V}(Y, X)
$$

$$
g_{V}\left(R^{V}(X, Y) Z, W\right)+g_{V}\left(R^{V}(X, Y) W, Z\right)=2 B^{V}(X, Y, Z, W),
$$

where

$$
\begin{aligned}
& B^{V}(X, Y, Z, W)= \\
& \quad \nabla_{Y}^{V} C_{V}\left(\nabla_{X}^{V} V, Z, W\right)-\nabla_{X}^{V} C_{V}\left(\nabla_{Y}^{V} V, Z, W\right)+C_{V}\left(R^{V}(Y, X) V, Z, W\right) ;
\end{aligned}
$$


(iii)

$$
R^{V}(X, Y) Z+R^{V}(Y, Z) X+R^{V}(Z, X) Y=0
$$

$$
\begin{aligned}
& g_{V}\left(R^{V}(X, Y) Z, W\right)-g_{V}\left(R^{V}(Z, W) X, Y\right)= \\
& B^{V}(Z, Y, X, W)+B^{V}(X, Z, Y, W)+B^{V}(W, X, Z, Y) \\
& +B^{V}(Y, W, Z, X)+B^{V}(W, Z, X, Y)+B^{V}(X, Y, Z, W) \text {. }
\end{aligned}
$$

We can also define the Jacobi operator $R^{\gamma}$ along an $L$-admissible curve $\gamma:[a, b] \subset$ $\mathbb{R} \rightarrow M$. Recall that the curve $\gamma$ is $L$-admissible if $\dot{\gamma}$ belongs to $A$ and consider a smooth variation $\Lambda:[a, b] \times(-\varepsilon, \varepsilon) \rightarrow M$, which is a two-parameter map. Given $s_{0} \in(-\varepsilon, \varepsilon)$ and $t_{0} \in[a, b]$, we will denote by $\gamma_{s_{0}}:[a, b] \rightarrow M$ the curve defined as $\gamma_{s_{0}}(t)=\Lambda\left(t, s_{0}\right)$ for every $t \in[a, b]$ and by $\beta_{t_{0}}:(-\varepsilon, \varepsilon) \rightarrow M$ the curve defined as $\beta_{t_{0}}(s)=\Lambda\left(t_{0}, s\right)$ for every $s \in(-\varepsilon, \varepsilon)$, which are the longitudinal and the transverse curves of the variation, respectively. Moreover, we will use the notation $\Lambda_{t}(t, s)=$ $\dot{\gamma}_{s}(t)$ and $\Lambda_{s}(t, s)=\dot{\beta}_{t}(s)$ and we will denote by $\Lambda^{*}(T M)$ the vector bundle over $[a, b] \times(-\varepsilon, \varepsilon)$ induced by $\pi: T M \rightarrow M$ through $\Lambda$. Then the space of smooth sections of $\Lambda^{*}(T M)$ will be denoted as $\mathfrak{X}(\Lambda)$. Observe that a vector field $V \in \mathfrak{X}(\Lambda)$ induces vector fields in $\mathfrak{X}\left(\gamma_{s_{0}}\right)$ and $\mathfrak{X}\left(\beta_{t_{0}}\right)$ for every $s_{0} \in(-\varepsilon, \varepsilon)$ and $t_{0} \in[a, b]$. We will say that $V$ is $L$-admissible if $V(t, s) \in A$ for every $(t, s) \in[a, b] \times(-\varepsilon, \varepsilon)$. When $\Lambda$ lies in the domain of a coordinate system $x^{1}, \ldots, x^{n}$, we will denote $\Lambda^{i}=x^{i} \circ \Lambda$. Notice that when we have a variation of curves (or more generally a two parameters map), as the Chern connection is free of torsion, we have the following property:

$$
D_{\gamma_{s}}^{V} \dot{\beta}_{t}=D_{\beta_{t}}^{V} \dot{\gamma}_{s}
$$

(see also [15, Proposition 3.2]). We say that the variation $\Lambda$ is $L$-admissible if $\gamma_{s}$ is $L$-admissible for every $s \in(-\varepsilon, \varepsilon)$. Moreover, we will denote by $W$ the variational vector field of $\Lambda$ along $\gamma$, namely, $W(t)=\Lambda_{s}(t, 0)$ for every $t \in[a, b]$. If $\Lambda$ is $L$-admissible and $Z \in \mathfrak{X}(\Lambda)$, we can define

$$
R^{\Lambda}(Z):=D_{\gamma_{s}}^{\Lambda_{t}} D_{\beta_{t}}^{\Lambda_{t}} Z-D_{\beta_{t}}^{\Lambda_{t}} D_{\gamma_{s}}^{\Lambda_{t}} Z
$$

which is a smooth vector field along $\Lambda$. Now observe that given an $L$-admissible curve $\gamma:[a, b] \rightarrow M$, and an arbitrary smooth vector field $W$ along $\gamma$ there always exists a (non-unique) $L$-admissible variation $\Lambda$ of $\gamma$ with $W$ as variational vector field. In fact, it is well-known that we can choose a variation $\Lambda:[a, b] \times(-\varepsilon, \varepsilon) \rightarrow M$ of $\gamma$ having $W$ as a variation vector field. As $\Lambda$ is at least $C^{1}$, being $A$ an open subset and $[a, b]$ compact, we can choose a smaller $\varepsilon$ if necessary in such a way that $\Lambda$ is $L$-admissible. Moreover, following [16], we can define

$$
R^{\gamma}(\dot{\gamma}, W) Z:=R^{\Lambda}(\tilde{Z}),
$$

where $Z$ is a smooth vector field along $\gamma$ and $\tilde{Z}$ is a smooth extension of $Z$ to $\Lambda$. As was proven in [16], the operator $R^{\gamma}$ is well-defined because it does not depend on the choice of the variation $\Lambda$ neither on the extension of $Z$.

In general, $R^{\gamma}(\dot{\gamma}, W) Z$ is not tensorial in $W$, since it is not $C^{\infty}([a, b])$-linear in $W$, but as a consequence of [16, Corollary 1.3], it is when $Z=\dot{\gamma}$. Moreover, when $\gamma$ is a geodesic

$$
R^{\gamma}(\dot{\gamma}, W) \dot{\gamma}=R_{\dot{\gamma}}(\dot{\gamma}, W) \dot{\gamma}
$$


where we denote by $R_{v}$ the Chern curvature (see [16] or [4, Formula (3.3.2) and Exercise 3.9.6]). Finally, for any $v \in A$ and $w \in T_{\pi(v)} M$, the flag curvature can be computed as

$$
K_{v}(w)=\frac{g_{v}\left(R^{\gamma_{v}}\left(\dot{\gamma}_{v}, W\right) W\left(t_{0}\right), v\right)}{L(v) g_{v}(w, w)-g_{v}(v, w)^{2}},
$$

where $\gamma_{v}$ is the geodesic such that $\dot{\gamma}_{v}\left(t_{0}\right)=v$ and $W$ is a smooth vector field along $\gamma$ such that $W\left(t_{0}\right)=w($ see $[16$, Remark 2.3]).

\section{VARIATION OF THE ENERGY}

Given a pseudo-Finsler manifold $(M, L)$, we shall denote by $C_{L}(M,[a, b])$ the space of $L$-admissible piecewise smooth curves in $M$ defined on the closed interval $[a, b]$. We write $T_{\gamma} C_{L}(M,[a, b])$ for the set of all $L$-admissible, piecewise smooth continuous vector fields along $\gamma \in C_{L}(M,[a, b])$ with the same breaks as $\gamma$. The energy functional on $C_{L}(M,[a, b])$ is the function

$$
E: \gamma \in C_{L}(M,[a, b]) \mapsto E(\gamma):=\frac{1}{2} \int_{b}^{a} L \circ \dot{\gamma} d t \in \mathbb{R} .
$$

We are going to show that the geodesics of $(M, L)$ are the critical points of $E$. To do this, we calculate the first and second variation formula for $E$.

Let $\gamma \in C_{L}(M,[a, b])$, and consider the piecewise smooth variation $\Lambda:[a, b] \times$ $(-\varepsilon, \varepsilon) \rightarrow M,(t, s) \mapsto \Lambda(t, s)$ of $\gamma$ with breaks $t_{0}:=a<t_{1}<t_{2}<\cdots<t_{h}<$ $t_{h+1}:=b$ and recall the notation for variations of the last section. Let us recall the definition of the Legendre transformation of a pseudo-Finsler metric, namely, the map $\mathscr{L}_{L}: A \rightarrow T M^{*}$, where $\mathscr{L}_{L}(v)$ is defined as the one-form given by $\mathscr{L}_{L}(v)(w)=$ $g_{v}(v, w)$ for every $w \in T_{\pi(v)} M$ and $g$ is the fundamental tensor of $L$.

Proposition 3.1. Keeping the notation introduced above, let $\Lambda$ be an $L$-admissible piecewise smooth variation of $\gamma$. Then we have the first variation formula

$$
\begin{aligned}
E^{\prime}(0):= & \left.\frac{d\left(E\left(\gamma_{s}\right)\right)}{d s}\right|_{s=0} \\
=- & \int_{a}^{b} g_{\dot{\gamma}}\left(W, D_{\gamma}^{\dot{\gamma}} \dot{\gamma}\right) d t+\left.g_{\dot{\gamma}}(W, \dot{\gamma})\right|_{a} ^{b} \\
& \quad+\sum_{i=1}^{h}\left(\mathcal{L}_{L}\left(\dot{\gamma}\left(t_{i}^{+}\right)\right)\left(W\left(t_{i}\right)\right)-\mathcal{L}_{L}\left(\dot{\gamma}\left(t_{i}^{-}\right)\right)\left(W\left(t_{i}\right)\right)\right),
\end{aligned}
$$

where $\dot{\gamma}\left(t_{i}^{+}\right)$(resp. $\dot{\gamma}\left(t_{i}^{-}\right)$) denote the right (resp. left) velocity at the breaks.

Proof. As the variation is piecewise smooth, we get

$$
\begin{aligned}
\frac{d}{d s} E\left(\gamma_{s}\right) & =\frac{1}{2} \int_{a}^{b} \frac{d}{d s} g_{\dot{\gamma}_{s}}\left(\dot{\gamma}_{s}, \dot{\gamma}_{s}\right) d t \\
& =\int_{a}^{b}\left(g_{\dot{\gamma}_{s}}\left(D_{\beta_{t}}^{\dot{\gamma}_{s}} \dot{\gamma}_{s}, \dot{\gamma}_{s}\right)+C_{\dot{\gamma}_{s}}\left(D_{\beta_{t}}^{\dot{\gamma}_{s}} \dot{\gamma}_{s}, \dot{\gamma}_{s}, \dot{\gamma}_{s}\right)\right) d t \\
& =\int_{a}^{b} g_{\dot{\gamma}_{s}}\left(D_{\gamma_{s}}^{\dot{\gamma}_{s}} \dot{\beta}_{t}, \dot{\gamma}_{s}\right) d t,
\end{aligned}
$$


where we have used first that the Chern connection is almost $g$-compatible and then Remark 2.8 and (7). Moreover, applying again that the Chern connection is almost $g$-compatible, we find that

$$
g_{\dot{\gamma}}\left(D_{\gamma}^{\dot{\gamma}} W, \dot{\gamma}\right)=\frac{d}{d t}\left(g_{\dot{\gamma}}(W, \dot{\gamma})\right)-g_{\dot{\gamma}}\left(W, D_{\gamma}^{\dot{\gamma}} \dot{\gamma}\right),
$$

because $C_{\dot{\gamma}}\left(D_{\gamma}^{\dot{\gamma}} \dot{\gamma}, W, \dot{\gamma}\right)=0$ (again by Remark 2.8). Substituting (12) in (11) with $s=0$ and integrating, we get finally (10).

Proposition 3.1 allows us to define formally the differential of $E$ in $\gamma$ as the map $d E_{\gamma}: T_{\gamma} C(M,[a, b]) \rightarrow \mathbb{R}$ given by

$$
\begin{aligned}
d E_{\gamma}(W)=-\int_{a}^{b} g_{\dot{\gamma}}\left(W, D_{\gamma}^{\dot{\gamma}} \dot{\gamma}\right) d t & +\left[g_{\dot{\gamma}}(W, \dot{\gamma})\right]_{a}^{b} \\
& +\sum_{i=1}^{h}\left(\mathscr{L}_{L}\left(\dot{\gamma}\left(t_{i}^{+}\right)\right)\left(W\left(t_{i}\right)\right)-\mathscr{L}_{L}\left(\dot{\gamma}\left(t_{i}^{-}\right)\right)\left(W\left(t_{i}\right)\right)\right),
\end{aligned}
$$

for any $W \in T_{\gamma} C_{L}(M,[a, b])$.

From now on, given a smooth $L$-admissible curve $\gamma:[a, b] \rightarrow M$ and $W \in \mathfrak{X}(\gamma)$, we write $W^{\prime}=D_{\gamma}^{\dot{\gamma}} W$. As we shall see later, the critical points of the energy functional are geodesics when some boundary conditions are imposed. Now we compute the second variation formula.

Proposition 3.2. Let $\gamma:[a, b] \rightarrow M$ be a geodesic of $(M, L)$ and consider an $L$-admissible smooth variation $\Lambda$. Then with the above notation

$$
\begin{aligned}
E^{\prime \prime}(0) & =\left.\frac{d^{2}}{d s^{2}} E\left(\gamma_{s}\right)\right|_{s=0} \\
& =\int_{a}^{b}\left(-g_{\dot{\gamma}}\left(R^{\gamma}(\dot{\gamma}, W) W, \dot{\gamma}\right)+g_{\dot{\gamma}}\left(W^{\prime}, W^{\prime}\right)\right) d t+\left[g_{\dot{\gamma}}\left(\left.D_{\beta_{t}}^{\dot{\gamma}} \dot{\beta}_{t}\right|_{s=0}, \dot{\gamma}\right)\right]_{a}^{b},
\end{aligned}
$$

where $\left.D_{\beta_{t}}^{\dot{\gamma}} \dot{\beta}_{t}\right|_{s=0}$ is the transverse acceleration vector field (cf. [25, page 266]) of the variation.

Proof. We will use Remark 2.8 along the proof without further comment. Using (11) and the almost $g$-compatibility of the Chern connection, we get

$$
\begin{aligned}
\frac{d^{2}}{d s^{2}} E\left(\gamma_{s}\right)=\frac{d}{d s} \int_{a}^{b} g_{\dot{\gamma}_{s}}\left(D_{\gamma_{s}}^{\dot{\gamma}_{s}} \dot{\beta}_{t}, \dot{\gamma}_{s}\right) d t=\int_{a}^{b} \frac{d}{d s} g_{\dot{\gamma}_{s}}\left(D_{\gamma_{s}}^{\dot{\gamma}_{s}} \dot{\beta}_{t}, \dot{\gamma}_{s}\right) d t \\
=\int_{a}^{b}\left(g_{\dot{\gamma}_{s}}\left(D_{\beta_{t}}^{\dot{\gamma}_{s}} D_{\gamma_{s}}^{\dot{\gamma}_{s}} \dot{\beta}_{t}, \dot{\gamma}_{s}\right)+g_{\dot{\gamma}_{s}}\left(D_{\gamma_{s}}^{\dot{\gamma}_{s}} \dot{\beta}_{t}, D_{\beta_{t}}^{\dot{\gamma}_{s}} \dot{\gamma}_{s}\right)\right) d t .
\end{aligned}
$$

Since $D_{\beta_{t}}^{\dot{\gamma}_{s}} D_{\gamma_{s}}^{\dot{\gamma}_{s}} \dot{\beta}_{t}=D_{\gamma_{s}}^{\dot{\gamma}_{s}} D_{\beta_{t}}^{\dot{\gamma}_{s}} \dot{\beta}_{t}-R^{\gamma_{s}}\left(\dot{\gamma}_{s}, \dot{\beta}_{t}\right) \dot{\beta}_{t}($ see $(8))$,

$$
\begin{aligned}
\frac{d^{2}}{d s^{2}} E\left(\gamma_{s}\right)=\int_{a}^{b} g_{\dot{\gamma}_{s}}\left(D_{\gamma_{s}}^{\dot{\gamma}_{s}} D_{\beta_{t}}^{\dot{\gamma}_{s}} \dot{\beta}_{t}-R^{\gamma_{s}}\left(\dot{\gamma}_{s}, \dot{\beta}_{t}\right) \dot{\beta}_{t}, \dot{\gamma}_{s}\right) d t & \\
& +\int_{a}^{b} g_{\dot{\gamma}_{s}}\left(D_{\gamma_{s}}^{\dot{\gamma}_{s}} \dot{\beta}_{t}, D_{\beta_{t}}^{\dot{\gamma}_{s}} \dot{\gamma}_{s}\right) d t .
\end{aligned}
$$

Finally, as $\gamma_{0}=\gamma$ is a geodesic, we have $g_{\dot{\gamma}_{s}}\left(D_{\gamma_{s}}^{\dot{\gamma}_{s}} D_{\beta_{t}}^{\dot{\gamma}_{s}} \dot{\beta}_{t}, \dot{\gamma}_{s}\right)=\frac{d}{d t}\left(g_{\dot{\gamma}_{s}}\left(D_{\beta_{t}}^{\dot{\gamma}_{s}} \dot{\beta}_{t}, \dot{\gamma}_{s}\right)\right)$ for $s=0$, and using this in (14), integrating and recalling (7), we get (13). 
Observe that the transverse acceleration $\left.D_{\beta_{t}}^{\dot{\gamma}} \dot{\beta}_{t}\right|_{s=0}$ depends not only on the vector field $W$ along $\gamma$, but also on the variation $\Lambda$. We will see later that the dependence on the variation disappears when we put certain boundary conditions.

3.1. Submanifolds and second fundamental form. We refer the reader to [25] for the basic notions and notation on submanifolds in semi-Riemannian manifolds. Let us assume that $(M, L)$ is a pseudo-Finsler manifold and $P \subset M$ a submanifold of $M$. We denote the tangent bundle of $P$ as TP and define the normal bundle $T P^{\perp}$ of $P$ as the set of vectors $v \in A$ such that $\pi(v) \in P$ and $g_{v}(v, w)=0$ for every $w \in T_{\pi(v)} P$. We write $T_{p} P^{\perp}=T P^{\perp} \cap T_{p} M$ for every $p \in P$. This is a conic subset, namely, if $v \in T_{p} P^{\perp}$, then $\lambda v \in T_{p} P^{\perp}$ for every $\lambda>0$. Notice that even though $T P^{\perp}$ is not necessarily a fiber bundle over $P$, it admits a structure of submersion. By abuse of notation, we write also $\pi$ for the restriction to $T P^{\perp}$ of the natural projection $\pi: T M \rightarrow M$. Let $P_{0}:=\left\{p \in P: \exists v \in T P^{\perp}, \pi(v)=p\right\}, r:=\operatorname{dim} P$, and recall that $n=\operatorname{dim} M$.

Lemma 3.3. If $T P^{\perp}$ is not empty, then it is an $n$-dimensional submanifold of $T M$. The subset $P_{0}$ is open in $P$, and the map $\pi: T P^{\perp} \rightarrow P_{0}$ is a submersion. In particular, for every $p \in P, T_{p} P^{\perp}$ is a submanifold of $T_{p} M$ of dimension $n-r$.

Proof. Assume that $E_{1}, \ldots, E_{r}$ is a local frame field over an open subset $\Xi$ of $P$, and observe that $A \cap \pi(\Xi)^{-1}$ is a submanifold of $T M$ of dimension $n+r$. Define $\varphi: A \cap \pi(\Xi)^{-1} \rightarrow \mathbb{R}^{r}$ as $\varphi(v)=\left(g_{v}\left(v, E_{1}\right), \ldots, g_{v}\left(v, E_{r}\right)\right)$. Observe that if $h:(-\epsilon, \epsilon) \rightarrow T_{p} M$ is a curve such that $h(0)=v$ and $\dot{h}(0)=u$ and $w \in T_{p} M$, then using the covariant derivative along the constant curve equal to $p,(3)$ and Remark 2.8 , we get

$$
\left.\frac{d}{d t} g_{h(t)}(h(t), w)\right|_{t=0}=g_{v}(u, w)+2 C_{v}(u, v, w)=g_{v}(u, w)
$$

and then the fiber derivative of $\varphi$ is given by $D_{f} \varphi_{v}(u)=\left(g_{v}\left(u, E_{1}\right), \ldots, g_{v}\left(u, E_{r}\right)\right)$ for every $u \in T_{\pi(v)} M$. As $g_{v}$ is a non-degenerate scalar product and $\left(E_{1}, \ldots, E_{r}\right)$ is linearly independent, this ensures that the map $\varphi$ is a submersion. Then $T P^{\perp} \cap \pi^{-1}(\Xi)=\varphi^{-1}(0)$, which, if not empty, is an $n$-dimensional submanifold of $T M$, and $T P^{\perp} \cap T_{p} M$ is a submanifold of $T_{p} M$ of dimension $n-r$. This implies that $P_{0}=\pi\left(T P^{\perp}\right)$ is open in $P$ and that $\pi: T P^{\perp} \rightarrow P_{0}$ is a submersion.

We denote by $\mathcal{F}(P)$ the space of smooth real functions on $P$, by $\mathfrak{X}(P)$ the $\mathcal{F}(P)$ module space of smooth sections of the fiber bundle $T P$ over $P$ and by $\mathfrak{X}(P)^{\perp}$ the space of smooth sections of $\pi: T P^{\perp} \rightarrow P_{0}$. Given $N \in \mathfrak{X}(P)^{\perp}$, we denote by $\mathfrak{X}(P) \stackrel{\perp}{N}$ the subset of smooth sections $W$ of $\pi: i^{*}(T M) \rightarrow P$ (where $i^{*}(T M)$ is the pull-back of $T M$ along the inclusion $i: P \rightarrow M)$ such that, for every $p \in P$, $W(p)$ is $g_{N}$-orthogonal to $T_{p} P$. Observe that in particular $N \in \mathfrak{X}(P)_{N}$. Then if $\left.g_{N}\right|_{T_{p} P \times T_{p} P}$ is nondegenerate, we have the decomposition

$$
T_{p} M=T_{p} P \oplus\left(T_{p} P\right)_{N}^{\perp},
$$

where $\left(T_{p} P\right)_{N} \stackrel{\perp}{N}$ is the subspace of $T_{p} M$ consisting of $g_{N}$-orthogonal vectors to $T_{p} P$. Then for every smooth section $V$ of $\pi: T M \rightarrow P$, we can define $\tan _{N}(V)$ (resp. $\operatorname{nor}_{N}(V)$ ) as the vector field in $\mathfrak{X}(P)$ obtained by projecting $V(p)$ to $T_{p} P$ (resp. $\left.\left(T_{p} P\right)_{N}^{\perp}\right)$, for every $p \in P$, through the decomposition (15).

Definition 3.4. Fix $N \in \mathfrak{X}(P)^{\perp}$ and suppose that $\left.g_{N}\right|_{T_{p} P \times T_{p} P}$ is nondegenerate for every $p \in P$. Then 
(i) the second fundamental form of $P$ in the direction of $N$ is the map $S_{N}^{P}: \mathfrak{X}(P) \times \mathfrak{X}(P) \rightarrow \mathfrak{X}(P) \frac{\perp}{N}$ given by $S_{N}^{P}(U, W)=\operatorname{nor}_{N} \nabla_{U}^{N} W$

(ii) the normal second fundamental form $\tilde{S}_{N}^{P}: \mathfrak{X}(P) \rightarrow \mathfrak{X}(P)$ is given by $\tilde{S}_{N}^{P}(U)=\tan _{N} \nabla_{U}^{N} N$.

Proposition 3.5. With the above notation, $S_{N}^{P}$ is $\mathcal{F}(P)$-bilinear and symmetric and $\tilde{S}_{N}^{P}$ is $\mathcal{F}(P)$-linear. Moreover,

$$
g_{N}\left(S_{N}^{P}(U, W), N\right)=-g_{N}\left(\tilde{S}_{N}^{P}(U), W\right)
$$

for every $U, W \in \mathfrak{X}(P)$.

Proof. First we show that $S_{N}^{P}$ is $\mathcal{F}(P)$-bilinear. This is immediate for the first variable. As to the second one, let $f \in \mathcal{F}(P)$ and $U, W \in \mathfrak{X}(P)$. Then

$$
S_{N}^{P}(U, f W)=\operatorname{nor}_{N} \nabla_{U}^{N}(f W)=\operatorname{nor}_{N}\left(U(f) W+f \nabla_{U}^{N}(W)\right)=f \operatorname{nor}_{N}\left(\nabla_{U}^{N} W\right),
$$

since $W$ is tangent to $P$. For the symmetry,

$$
S_{N}^{P}(U, W)-S_{N}^{P}(W, U)=\operatorname{nor}_{N}\left(\nabla_{U}^{N} W-\nabla_{W}^{N} U\right)=\operatorname{nor}_{N}[U, W]=0,
$$

since $[U, W]$ is tangent to $P$. Again it is straightforward to check that $\tilde{S}_{N}^{P}$ is $\mathcal{F}(P)$-linear. For (16), using that the Chern connection is almost $g$-compatible, $g_{N}(N, W)=0$ and Remark 2.8, we get

$$
\begin{aligned}
g_{N}\left(S_{N}^{P}(U, W), N\right) & =g_{N}\left(\nabla_{U}^{N} W, N\right) \\
& =-g_{N}\left(W, \nabla_{U}^{N} N\right)-2 C_{N}\left(\nabla_{U}^{N} N, W, N\right)=-g_{N}\left(\tilde{S}_{N}^{P}(U), W\right),
\end{aligned}
$$

as required.

Remark 3.6. Observe that from the homogeneity of the Chern connection it follows that $S_{\lambda N}^{P}=S_{N}^{P}$, and then from (16), that $\tilde{S}_{\lambda N}^{P}=\lambda \tilde{S}_{N}^{P}$. Moreover, we shall interpret $S_{N}^{P}$ and $\tilde{S}_{N}^{P}$ as maps $S_{N}^{P}: T_{p} P \times T_{p} P \rightarrow\left(T_{p} P\right)_{N}^{\perp}$ and $\tilde{S}_{N}^{P}: T_{p} P \rightarrow T_{p} P$, respectively, even when $\mathfrak{X}(P)^{\perp}$ is empty (think that $P$ could be non-orientable), since if $T_{p} P^{\perp}$ is not empty, then there is some open subset $\Xi \subset P$ such that $\mathfrak{X}(\Xi)^{\perp}$ is not empty.

3.2. The endmanifold case. Consider now the space of curves

$$
C_{L}(P, Q) \subset C_{L}(M,[a, b])
$$

joining two submanifolds $P$ and $Q$ of $M$, namely,

$$
C_{L}(P, Q):=\left\{\gamma \in C_{L}(M,[a, b]): \gamma(a) \in P, \gamma(b) \in Q\right\} .
$$

When we consider a piecewise smooth $(P, Q)$-variation of $\gamma \in C_{L}(P, Q)$ by curves in $C_{L}(P, Q)$, the variational vector field is tangent to $P$ and $Q$ at the endpoints. Indeed, we define

$$
T_{\gamma} C_{L}(P, Q)=\left\{W \in T_{\gamma} C_{L}(M,[a, b]): W(a) \in T_{\gamma(a)} P, W(b) \in T_{\gamma(b)} Q\right\} .
$$

Moreover, we say that $\gamma$ is a critical point of $\left.E\right|_{C_{L}(P, Q)}$ if $d E_{\gamma}(W)=0$ for every $W \in T_{\gamma} C_{L}(P, Q)$.

Corollary 3.7. Let $\gamma \in C_{L}(P, Q)$ and assume that the Legendre transformation $\mathscr{L}_{L}$ is injective. Then $\gamma$ is a critical point of the energy functional $\left.E\right|_{C_{L}(P, Q)}$ if and only if $\gamma$ is a geodesic $g_{\dot{\gamma}}$-orthogonal to $P$ and $Q$. 
Proof. Let $t_{0} \in(a, b)$ an instant where $\gamma$ is smooth. As the scalar product $g_{\dot{\gamma}}$ is nondegenerate, if we assume that $D_{\gamma}^{\dot{\gamma}} \dot{\gamma} \neq 0$, using bump functions, we can find a vector field $W$ such that $g_{\dot{\gamma}}\left(D_{\gamma}^{\dot{\gamma}} \dot{\gamma}, W\right)>0$ in a neighborhood of $t_{0}$ that does not contain breaks and is zero everywhere. Then using (10), we get a contradiction. Thus $\gamma$ must be a piecewise geodesic. Assume that $\dot{\gamma}\left(t_{i}^{+}\right) \neq \dot{\gamma}\left(t_{i}^{-}\right)$for some $i=$ $1, \ldots, h$. As $\mathscr{L}_{L}$ is assumed to be injective, we can find a variational vector field $W_{i}$ such that $\mathscr{L}_{L}\left(\dot{\gamma}\left(t_{i}^{+}\right)\right)\left(W_{i}\right)-\mathscr{L}_{L}\left(\dot{\gamma}\left(t_{i}^{-}\right)\right)\left(W_{i}\right) \neq 0$ and it is zero at the other breaks. This gives a contradiction in (10), since $\gamma$ is a critical point. Therefore, $\gamma$ is a geodesic. Finally given $w \in T_{\gamma(a)} P$, construct a vector field $W$ such that $W(a)=w$ and $W(b)=0$. Then (10) implies that $g_{\dot{\gamma}(a)}(\dot{\gamma}(a), w)=0$. Analogously, we can show that for any $v \in T_{\gamma(b)} Q, g_{\dot{\gamma}(b)}(\dot{\gamma}(b), v)=0$. The converse is trivial.

Corollary 3.8. Let $\gamma \in C_{L}(P, Q)$ be a geodesic of $(M, L)$ that is $g_{\dot{\gamma}}$-orthogonal to $P$ and $Q$ at the endpoints and such that $\left.g_{\dot{\gamma}(a)}\right|_{P \times P}$ and $\left.g_{\dot{\gamma}(b)}\right|_{Q \times Q}$ are nondegenerate. Consider a smooth L-admissible $(P, Q)$-variation. Then

$$
\begin{aligned}
E^{\prime \prime}(0)=\int_{a}^{b}\left(-g_{\dot{\gamma}}\left(R^{\gamma}(\dot{\gamma}, W) W, \dot{\gamma}\right)+g_{\dot{\gamma}}\left(W^{\prime}, W^{\prime}\right)\right) d t & \\
& +g_{\dot{\gamma}(b)}\left(S_{\dot{\gamma}(b)}^{P}(W, W), \dot{\gamma}(b)\right)-g_{\dot{\gamma}(a)}\left(S_{\dot{\gamma}(a)}^{Q}(W, W), \dot{\gamma}(a)\right),
\end{aligned}
$$

where $W$ is the variational vector field of the variation along $\gamma$.

Proof. It is a straightforward consequence of the definition of the second fundamental form in Definition 3.4 and (13).

3.3. The index form. When $\gamma$ is a geodesic of a pseudo-Finsler manifold $(M, L)$ such that it is $g_{\dot{\gamma}}$-orthogonal to $P$ and $Q$ at the endpoints and such that $\left.g_{\dot{\gamma}(a)}\right|_{P \times P}$ and $\left.g_{\dot{\gamma}(b)}\right|_{Q \times Q}$ are nondegenerate, we can define the $(P, Q)$-index form of $\gamma$ as

$$
\begin{aligned}
I_{P, Q}^{\gamma}(V, W)=\int_{a}^{b}\left(-g_{\dot{\gamma}}\left(R^{\gamma}(\dot{\gamma}, V) W, \dot{\gamma}\right)+g_{\dot{\gamma}}\left(V^{\prime}, W^{\prime}\right)\right) d t \\
\quad+g_{\dot{\gamma}(b)}\left(S_{\dot{\gamma}(b)}^{P}(V, W), \dot{\gamma}(b)\right)-g_{\dot{\gamma}(a)}\left(S_{\dot{\gamma}(a)}^{Q}(V, W), \dot{\gamma}(a)\right),
\end{aligned}
$$

where $V, W \in T_{\gamma} C_{L}(P, Q)$.

Remark 3.9. Observe that when $P$ (resp. $Q$ ) is a hypersurface of $M$ and $\gamma$ is orthogonal to $P$ (resp. to $Q$ ), the nondegeneracy condition on $\left.g_{\dot{\gamma}(a)}\right|_{P \times P}$ (resp. $\left.\left.g_{\dot{\gamma}(b)}\right|_{Q \times Q}\right)$ is equivalent to $L(\dot{\gamma}(a)) \neq 0$ (resp. $L(\dot{\gamma}(b)) \neq 0$ ).

Let us now give a useful property of the tensor $B^{V}$ defined in subsection 2.6.

Lemma 3.10. Let $(M, L)$ be a pseudo-Finsler manifold and $\Omega$ some open subset of $M$ and $V$ an $L$-admissible vector field in $\Omega$. Then for every $X, Y, Z, W \in \mathfrak{X}(\Omega)$, $B^{V}$ satisfies

(i) If $Z=V$ or $W=V$, then

$$
B^{V}(X, Y, Z, W)=0 .
$$

(ii) If at least two of the vector fields $X, Y, Z, W$ are equal to $V$, then

$$
B^{V}(X, Y, Z, W)=0 .
$$

(iii) For any curve $\gamma$ and $U, P \in \mathfrak{X}(\gamma)$,

$$
g_{\dot{\gamma}}\left(R^{\gamma}(\dot{\gamma}, U) \dot{\gamma}, P\right)=-g_{\dot{\gamma}}\left(R^{\gamma}(\dot{\gamma}, U) P, \dot{\gamma}\right)
$$


Proof. Since $B^{V}$ is symmetric in the last two arguments, to prove $(i)$ it is enough to show that $B^{V}(X, Y, V, W)=0$.

Using the definition of $B^{V}$ and Remark 2.8, we get

$$
B^{V}(X, Y, V, W)=\nabla_{Y}^{V} C_{V}\left(\nabla_{X}^{V} V, V, W\right)-\nabla_{X}^{V} C_{V}\left(\nabla_{Y}^{V} V, V, W\right) .
$$

Then, $(i)$ follows from the symmetry of the tensors $\nabla_{Y}^{V} C_{V}$ and $\nabla_{X}^{V} C_{V}$ together with relation (5), because

$$
\begin{aligned}
B^{V}(X, Y, V, W)=\nabla_{Y}^{V} C_{V} & \left(V, \nabla_{X}^{V} V, W\right)-\nabla_{X}^{V} C_{V}\left(V, \nabla_{Y}^{V} V, W\right)= \\
& -C_{V}\left(\nabla_{Y}^{V} V, \nabla_{X}^{V} V, W\right)+C_{V}\left(\nabla_{X}^{V} V, \nabla_{Y}^{V} V, W\right)=0 .
\end{aligned}
$$

Now, $(i i)$ is a simple consequence of $(i)$ and the antisymmetry of $B^{V}$ in the first two arguments.

(iii), on its turn, is simply a generalization of [16, Eq. (14)], stating that it is valid for any curve $\gamma$, not only for geodesics. The proof is exactly the same.

Proposition 3.11. The kernel of $I_{P, Q}^{\gamma}$ consists of the vector fields $V \in T_{\gamma} C_{L}(P, Q)$ satisfying

$$
V^{\prime \prime}=R^{\gamma}(\dot{\gamma}, V) \dot{\gamma} \quad, \quad \tan _{\dot{\gamma}} V^{\prime}(a)=\tilde{S}_{\dot{\gamma}(a)}^{P}(V(a)) \quad \text { and } \tan _{\dot{\gamma}} V^{\prime}(b)=\tilde{S}_{\dot{\gamma}(b)}^{Q}(V(b)) .
$$

Proof. Using the fact established in the above lemma, that $g_{\dot{\gamma}}\left(R^{\gamma}(\dot{\gamma}, V) W, \dot{\gamma}\right)=-g_{\dot{\gamma}}\left(R^{\gamma}(\dot{\gamma}, V) \dot{\gamma}, W\right)$, and taking into account that, since the Chern connection is almost $g$-compatible and $\gamma$ is a geodesic,

$$
g_{\dot{\gamma}}\left(V^{\prime}, W^{\prime}\right)=\frac{d}{d t}\left(g_{\dot{\gamma}}\left(V^{\prime}, W\right)\right)-g_{\dot{\gamma}}\left(V^{\prime \prime}, W\right),
$$

we may conclude that the index form can also be expressed as

$$
\begin{aligned}
I_{P, Q}^{\gamma}(V, W)=\int_{a}^{b}\left(g_{\dot{\gamma}}\left(R^{\gamma}(\dot{\gamma}, V) \dot{\gamma}, W\right)-g_{\dot{\gamma}}\left(V^{\prime \prime}, W\right)\right) d s+\left[g_{\dot{\gamma}}\left(V^{\prime}, W\right)\right]_{a}^{b} \\
\quad+g_{\dot{\gamma}(b)}\left(S_{\dot{\gamma}(b)}^{P}(V, W), \dot{\gamma}(b)\right)-g_{\dot{\gamma}(a)}\left(S_{\dot{\gamma}(a)}^{Q}(V, W), \dot{\gamma}(a)\right) .
\end{aligned}
$$

This means that $V \in T_{\gamma} C_{L}(P, Q)$ belongs to the kernel of the index form if and only if $V^{\prime \prime}-R^{\gamma}(\dot{\gamma}, V) \dot{\gamma}=0$ and

$$
\begin{array}{r}
g_{\dot{\gamma}(a)}\left(V^{\prime}(a), W(a)\right)+g_{\dot{\gamma}(a)}\left(S_{\dot{\gamma}(a)}^{P}(V, W), \dot{\gamma}(a)\right)=0, \\
g_{\dot{\gamma}(b)}\left(V^{\prime}(b), W(b)\right)+g_{\dot{\gamma}(b)}\left(S_{\dot{\gamma}(b)}^{Q}(V, W), \dot{\gamma}(b)\right)=0,
\end{array}
$$

at the endpoints. Using (16), we get that

$$
g_{\dot{\gamma}(a)}\left(V^{\prime}(a)-\tilde{S}_{\dot{\gamma}(a)}^{P}(V(a)), u\right)=0, \quad g_{\dot{\gamma}(b)}\left(V^{\prime}(b)-\tilde{S}_{\dot{\gamma}(b)}^{P}(V(b)), w\right)=0
$$

for every $u \in T_{\gamma(a)} P$ and $w \in T_{\gamma(b)} Q$. Thus $\tan _{\dot{\gamma}} V^{\prime}(a)=\tilde{S}_{\dot{\gamma}(a)}^{P}(V(a))$ and $\tan _{\dot{\gamma}} V^{\prime}(b)=\tilde{S}_{\dot{\gamma}(b)}^{Q}(V(b))$ as required. 


\subsection{Jacobi fields, Conjugate and focal points.}

Definition 3.12. Given a geodesic $\gamma:[a, b] \rightarrow M$ of $(M, L)$, a Jacobi field of $\gamma$ is a vector field $J$ along $\gamma$ satisfying

$$
J^{\prime \prime}=R^{\gamma}(\dot{\gamma}, J) \dot{\gamma}
$$

Moreover, given a submanifold $P$ such that $\gamma(a) \in P$ and $\dot{\gamma}(a)$ is $g_{\dot{\gamma}(a)}$-orthogonal to $P$, we say that a Jacobi field is $P$-Jacobi if $J(a)$ is tangent to $P$ and $\tan _{\dot{\gamma}} J^{\prime}(a)=$ $\tilde{S}_{\dot{\gamma}(a)}^{P}(J(a))$. An instant $t_{0} \in(a, b]$ is called

(i) conjugate if there exists a Jacobi field $J$ along $\gamma$ such that $J(a)=J\left(t_{0}\right)=0$,

(ii) $P$-focal if there exists a $P$-Jacobi field $J$ such that $J\left(t_{0}\right)=0$.

Given a geodesic $\gamma:[a, b] \rightarrow M$ of a pseudo-Finsler manifold, we can construct a parallel orthonormal frame field along $\gamma$. Indeed, fix an orthonormal basis $e_{1}, e_{2}, \ldots, e_{n}$ of $\left(T_{\gamma(a)} M, g_{\dot{\gamma}(a)}\right)$, namely, a basis satisfying $g_{\dot{\gamma}(a)}\left(e_{i}, e_{j}\right)=\varepsilon_{i} \delta_{i j}$, where $\varepsilon_{i}^{2}=1, \delta_{i j}$ is the Kronecker's delta and $i, j=1, \ldots, n$. Then define the parallel vector fields $E_{1}, E_{2}, \ldots, E_{n}$ along $\gamma$ such that $E_{i}(a)=e_{i}$ for every $i=1, \ldots, n$. The fact that $\gamma$ is a geodesic implies that $g_{\dot{\gamma}}\left(E_{i}, E_{j}\right)=\varepsilon_{i} \delta_{i j}$, since

$$
\left(g_{\dot{\gamma}}\left(E_{i}, E_{j}\right)\right)^{\prime}=g_{\dot{\gamma}}\left(E_{i}^{\prime}, E_{j}\right)+g_{\dot{\gamma}}\left(E_{i}, E_{j}^{\prime}\right)+2 C_{\dot{\gamma}}\left(D_{\gamma}^{\dot{\gamma}} \dot{\gamma}, E_{i}, E_{j}\right)=0,
$$

where we have used that the Chern connection is almost $g$-compatible, $\gamma$ is a geodesic and the frame field is parallel along $\gamma$.

We recall that a variation is called geodesic if its longitudinal curves are geodesics. As in the pseudo-Riemannian case, we have

Proposition 3.13. In any pseudo-Finsler manifold, the variation vector field of a geodesic variation is a Jacobi field.

We note that this is true also in the more general setting of spray manifolds, see [28, Section 8.2.2].

Lemma 3.14. Let $\gamma:[a, b] \rightarrow M$ be a geodesic of $(M, L)$ with $\gamma(a)=p$. Then for any $v, w \in T_{p} M$, there exists a unique Jacobi field such that $J(a)=v$ and $J^{\prime}(a)=w$.

Using a parallel orthonormal frame field along $\gamma$, the proof is the same as in the pseudo-Riemannian case; see, e.g., [25, page 217].

Proposition 3.15. Let $p$ be a point of a pseudo-Finsler manifold $(M, L)$. If $v \in$ $T_{p} M \cap A$ belongs to the domain of $\exp _{p}$, then for any $w \in T_{v}\left(T_{p} M\right)$ we have

$$
d \exp _{p}(v)[w]=J(1),
$$

where $J$ is the unique Jacobi field on $\gamma_{v}$ such that $J(0)=0$ and $J^{\prime}(0)=w$.

Proof. Consider the variation $\tilde{\Lambda}(t, s)=t(v+s w)$ for $0 \leq t \leq 1$ and $s$ small enough, and define

$$
\Lambda(t, s)=\exp _{p}(\tilde{\Lambda}(t, s))=\gamma_{v+s w}(t) .
$$

By Proposition 3.13, the variation vector field $J(t)=\Lambda_{s}(t, 0)(t \in[0,1])$ is a Jacobi field. Moreover, as the curve $s \rightarrow \Lambda(0, s)=p$ is constant, $J(0)=0$ and if we denote by $\beta$ the constant curve with value $p$ and write $\gamma_{s}=\gamma_{v+s w}$, from (7) we get

$$
J^{\prime}(0)=D_{\beta}^{\dot{\gamma}_{s}} \dot{\gamma}_{s}(0)=w,
$$

since $\dot{\gamma}_{s}(0)=v+s w$. 
Given a geodesic $\gamma:[a, b] \rightarrow M$ of a pseudo-Finsler manifold, let $\dot{\gamma}^{\perp}:=\left\{v \in T_{\dot{\gamma}} M: g_{\dot{\gamma}}(\dot{\gamma}, v)=0\right\}$. When $L \circ \dot{\gamma} \neq 0$, we have the decomposition

$$
T_{\dot{\gamma}} M=\operatorname{span}(\dot{\gamma}) \oplus \dot{\gamma}^{\perp} .
$$

Moreover, if $Y \in \mathfrak{X}(\gamma)$, let us denote by $\tan _{\gamma}(Y)$ and $\operatorname{nor}_{\gamma}(Y)$ the first and second projection in the decomposition (17), respectively.

Lemma 3.16. With the above notation, if $L \circ \dot{\gamma} \neq 0$, then $\left(\tan _{\gamma}(Y)\right)^{\prime}=\tan _{\gamma}\left(Y^{\prime}\right)$ and $\left(\operatorname{nor}_{\gamma}(Y)\right)^{\prime}=\operatorname{nor}_{\gamma}\left(Y^{\prime}\right)$.

Proof. It is enough to prove that $\left(\tan _{\gamma}(Y)\right)^{\prime}=\tan _{\gamma}\left(Y^{\prime}\right)$, since the other equality comes then from $Y=\tan _{\gamma}(Y)+\operatorname{nor}_{\gamma}(Y)$. Observe that $g_{\dot{\gamma}}(Y, \dot{\gamma})=g_{\dot{\gamma}}\left(\tan _{\gamma}(Y), \dot{\gamma}\right)$. Using that $\gamma$ is a geodesic and the Chern connection is almost $g$-compatible, we get that $g_{\dot{\gamma}}\left(Y^{\prime}, \dot{\gamma}\right)=g_{\dot{\gamma}}\left(\left(\tan _{\gamma}(Y)\right)^{\prime}, \dot{\gamma}\right)$. This concludes the proof because $g_{\dot{\gamma}}(\dot{\gamma}, \dot{\gamma})=$ $L(\dot{\gamma}) \neq 0$.

Lemma 3.17. Consider a vector field $J$ along a geodesic $\gamma:[a, b] \rightarrow M$. If $J$ is a Jacobi field, then:

(i) $J$ is tangent to $\gamma$ if and only if $J(t)=\left(a_{1} t+a_{2}\right) \dot{\gamma}(t)$ with $a_{1}, a_{2} \in \mathbb{R}$;

(ii) the following statements are equivalent:

(a) $g_{\dot{\gamma}}(\dot{\gamma}, J)=0$

(b) there exist real numbers $a, b$ such that

$$
g_{\dot{\gamma}(a)}(\dot{\gamma}(a), J(a))=g_{\dot{\gamma}(b)}(\dot{\gamma}(b), J(b))=0,
$$

(c) there exists a real number a such that

$$
g_{\dot{\gamma}(a)}(\dot{\gamma}(a), J(a))=g_{\dot{\gamma}(a)}\left(\dot{\gamma}(a), J^{\prime}(a)\right)=0 .
$$

Moreover, if $\gamma$ is nonnull, that is, $L(\dot{\gamma}) \neq 0$, then $J$ is a Jacobi field if and only if $\operatorname{nor}_{\gamma}(J)$ and $\tan _{\gamma}(J)$ are Jacobi fields.

Proof. For (i), observe that

$$
R^{\gamma}(\dot{\gamma}, \dot{\gamma}) \dot{\gamma}=0
$$

(see [16, Theorem 2.2] and take into account that $R^{V}$ is antisymmetric in the first two arguments). Then, for $J=f \dot{\gamma}$, the Jacobi equation reduces to $\frac{d^{2} f}{d t^{2}}=0$. To see (ii), observe that since $\gamma$ is a geodesic,

$$
\left(g_{\dot{\gamma}}(J, \dot{\gamma})\right)^{\prime \prime}=g_{\dot{\gamma}}\left(J^{\prime \prime}, \dot{\gamma}\right)=g_{\dot{\gamma}}\left(R^{\gamma}(\dot{\gamma}, J) \dot{\gamma}, \dot{\gamma}\right)=0,
$$

by part (iii) of Lemma 3.10. Hence $g_{\dot{\gamma}}(J, \dot{\gamma})=C_{1} t+C_{2}$, where $C_{1}$ and $C_{2}$ are real constants, and $g_{\dot{\gamma}}\left(J^{\prime}, \dot{\gamma}\right)=C_{1}$, thus (ii) follows. For the last statement, observe that $R^{\gamma}\left(\dot{\gamma}, \tan _{\gamma} J\right) \dot{\gamma}=0$ and then $R^{\gamma}(\dot{\gamma}, J) \dot{\gamma}=R^{\gamma}\left(\dot{\gamma}, \operatorname{nor}_{\gamma} J\right) \dot{\gamma}\left(\right.$ recall that $R^{\gamma}(\dot{\gamma}, W) \dot{\gamma}$ is tensorial in $W)$. Using again that $g_{\dot{\gamma}}\left(R^{\gamma}(\dot{\gamma}, J) \dot{\gamma}, \dot{\gamma}\right)=0$ and Lemma 3.16, the Jacobi equation splits into the two equations

$$
\left(\tan _{\gamma} J\right)^{\prime \prime}=0 \quad \text { and } \quad\left(\operatorname{nor}_{\gamma} J\right)^{\prime \prime}=R^{\gamma}\left(\dot{\gamma}, \operatorname{nor}_{\gamma} J\right) \dot{\gamma} .
$$

Finally, applying part $(i)$, we conclude the proof.

Proposition 3.18. If $J_{1}$ and $J_{2}$ are Jacobi fields along a geodesic $\gamma$, then the function $g_{\dot{\gamma}}\left(J_{1}, J_{2}^{\prime}\right)-g_{\dot{\gamma}}\left(J_{1}^{\prime}, J_{2}\right)$ is constant. 
Proof. Using that $\gamma$ is a geodesic, that the Chern connection is almost $g$-compatible and that both $J_{1}$ and $J_{2}$ satisfy the Jacobi equation, we obtain

$$
\begin{aligned}
\left(g_{\dot{\gamma}}\left(J_{1}, J_{2}^{\prime}\right)-g_{\dot{\gamma}}\left(J_{1}^{\prime}, J_{2}\right)\right)^{\prime}= & g_{\dot{\gamma}}\left(J_{1}^{\prime}, J_{2}^{\prime}\right)+g_{\dot{\gamma}}\left(J_{1}, J_{2}^{\prime \prime}\right) \\
& -g_{\dot{\gamma}}\left(J_{1}^{\prime}, J_{2}^{\prime}\right)-g_{\dot{\gamma}}\left(J_{1}^{\prime \prime}, J_{2}\right) \\
= & g_{\dot{\gamma}}\left(J_{1}, R^{\gamma}\left(\dot{\gamma}, J_{2}\right) \dot{\gamma}\right)-g_{\dot{\gamma}}\left(R^{\gamma}\left(\dot{\gamma}, J_{1}\right) \dot{\gamma}, J_{2}\right) .
\end{aligned}
$$

Let us see that the last quantity is zero. If one of the vector fields $J_{1}, J_{2}$ is proportional to $\dot{\gamma}$ in some interval, then it follows from (18) and (19). Otherwise, if both of them are linearly independent to $\dot{\gamma}$ you can choose extensions $V, U_{1}$ and $U_{2}$ of $\dot{\gamma}, J_{1}$ and $J_{2}$, respectively, such that $\left[U_{1}, V\right]=\left[U_{2}, V\right]=0$ and

$$
\begin{aligned}
g_{\dot{\gamma}}\left(J_{1}, R^{\gamma}\left(\dot{\gamma}, J_{2}\right) \dot{\gamma}\right)-g_{\dot{\gamma}}\left(R^{\gamma}\left(\dot{\gamma}, J_{1}\right) \dot{\gamma}, J_{2}\right) & \\
& g_{V}\left(U_{1}, R^{V}\left(V, U_{2}\right) V\right)-g_{V}\left(R^{V}\left(V, U_{1}\right) V, U_{2}\right)
\end{aligned}
$$

(see [16, Theorem 2.2]), but the last quantity is zero along $\gamma$ because of (6) and part (ii) of Lemma 3.10. By continuity, we conclude that (20) is zero everywhere.

3.5. Remarks about Morse theory. Let us observe that in principle, there should not be further obstructions to prove that geodesics of a pseudo-Finsler metric are critical points of the energy functional in a suitable infinite dimensional $H^{1}$ Sobolev space, for example, by generalizing the proof in [10, Proposition 2.1]. But in order to make Morse theory available, we need to overcome several problems. The first one is that the Palais-Smale condition only holds in general when the pseudo-Finsler metric is in fact a Finsler metric (with positive definite fundamental tensor), since it is well-known that Palais-Smale condition fails for semi-Riemannian metrics. The second problem is the differentiability of the energy function in the $H^{1}$ Sobolev space, because it is $C^{2}$ only when the pseudo-Finsler metric is semiRiemannian (see [2, Proposition 3.2] and [8]). This has been overcome in the case of Finsler metrics using that the energy functional is $C^{2}$ in the $C^{1}$-topology (see $[9,11])$. The third problem is that when $A$ is strictly contained in $T M \backslash \mathbf{0}$, the space of $L$-admissible curves can be non-complete, thus it seems interesting to study conditions of completeness in the pseudo-Finsler metric to guarantee the validity of the Morse theory as in [9, 11]. In [12] some results of geodesic connectedness of conic Finsler metrics are deduced using causality of spacetimes endowed with a Killing vector. In the general case, when the fundamental tensor is allowed to have any signature, Lemma 3.18 is the key point to develop a relation between the spectral flow of a certain path of operators and the Maslov index of conjugate points as in [27]. In the presence of a Killing vector field, more precise results have been obtained in the Lorentzian realm $[7,13,17]$ and it is expectable to get similar results for Lorentzian Finsler metrics.

\section{REFERENCES}

[1] A. B. Aazami and M. Á. Javaloyes, Penrose's singularity theorem in a Finsler spacetime, arXiv:1410.7595 [math.DG] (2014).

[2] A. Abbondandolo And M. Schwarz, A smooth pseudo-gradient for the Lagrangian action functional, Adv. Nonlinear Stud., 9 (2009), pp. 597-623.

[3] P. L. Antonelli, R. S. Ingarden, and M. Matsumoto, The theory of sprays and Finsler spaces with applications in physics and biology, vol. 58 of Fundamental Theories of Physics, Kluwer Academic Publishers Group, Dordrecht, 1993. 
[4] D. Bao, S.-S. Chern, And Z. Shen, An Introduction to Riemann-Finsler geometry, Graduate Texts in Mathematics, Springer-Verlag, New York, 2000.

[5] J. K. Bеєм, Indefinite Finsler spaces and timelike spaces, Canad. J. Math., 22 (1970), pp. 1035-1039.

[6] J. K. Beem and M. A. Kishta, On generalized indefinite Finsler spaces, Indiana Univ. Math. J., $23(1973 / 74)$, pp. 845-853.

[7] A. M. Candela, J. L. Flores, and M. SÁnchez, Global hyperbolicity and PalaisSmale condition for action functionals in stationary spacetimes, Adv. Math., 218 (2008), pp. $515-536$.

[8] E. CAPOnio, The index of a geodesic in a Randers space and some remarks about the lack of regularity of the energy functional of a Finsler metric, Acta Math. Acad. Paedagog. Nyházi. (N.S.), 26 (2010), pp. 265-274.

[9] E. Caponio, M. Á. Javaloyes, and A. Masiello, Morse theory of causal geodesics in a stationary spacetime via Morse theory of geodesics of a Finsler metric, Ann. Inst. H. Poincaré Anal. Non Linéaire, 27 (2010), pp. 857-876.

[10] E. Caponio, M. Á. Javaloyes, and A. Masiello, On the energy functional on Finsler manifolds and applications to stationary spacetimes, Math. Ann., 351 (2011), pp. 365-392.

[11] E. Caponio, M. Á. Javaloyes, and A. Masiello, Addendum to "Morse theory of causal geodesics in a stationary spacetime via Morse theory of geodesics of a Finsler metric", Ann. Inst. H. Poincaré Anal. Non Linéaire, 30 (2013), pp. 961-968.

[12] E. Caponio, M. Á. Javaloyes, and M. SÁnchez, Wind Finslerian structures: from Zermelo's navigation to the causality of spacetimes, arXiv:1407.5494 [math.DG].

[13] F. Giannoni and P. Piccione, An intrinsic approach to the geodesical connectedness of stationary Lorentzian manifolds, Comm. Anal. Geom., 7 (1999), pp. 157-197.

[14] M. Hashiguchi, On parallel displacements in Finsler spaces, J. Math. Soc. Japan, 10 (1958), pp. 365-379

[15] M. Á. Javaloyes, Chern connection of a pseudo-Finsler metric as a family of affine connections, Pub. Math. Debrecen, 84 (2014), pp. 29-43

[16] M. Á. Javaloyes, Corrigendum to "Chern connection of a pseudo-Finsler metric as a family of affine connections", 85 (2014), pp. 481-487.

[17] M. Á. Javaloyes, A. Masiello, and P. Piccione, Pseudo focal points along Lorentzian geodesics and Morse index, Adv. Nonlinear Stud., 10 (2010), pp. 53-82.

[18] M. Á. Javaloyes and M. SÁnchez, On the definition and examples of Finsler metrics, Ann. Sc. Norm. Super. Pisa Cl. Sci. (5) Vol. XIII (2014), 813-858.

[19] M. Á. Javaloyes And H. VitóRIo, Zermelo navigation in pseudo-Finsler metrics, arXiv:1412.0465 [math.DG] (2014).

[20] R. L. Lovas, J. PÉK ANd J. Szilasi Ehresmann connections, metrics and good metric derivatives, in Finsler Geometry, Sapporo 2005, Advanced Studies in Pure Mathematics 48, Math. Soc. Japan, 2007, pp. 263-308

[21] H.-H. Matthias, Zwei Verallgemeinerungen eines Satzes von Gromoll und Meyer, Bonner Mathematische Schriften [Bonn Mathematical Publications], 126, Universität Bonn Mathematisches Institut, Bonn, 1980. Dissertation, Rheinische Friedrich-WilhelmsUniversität, Bonn, 1980.

[22] E. MinguzZi And M. SÁnchez, The causal hierarchy of spacetimes, in Recent developments in pseudo-Riemannian geometry, ESI Lect. Math. Phys., Eur. Math. Soc., Zürich, 2008, pp. 299-358.

[23] R. Miron, Metrical Finsler structures and metrical Finsler connections, J. Math. Kyoto Univ., 23 (1983), pp. 219-224

[24] A. MoóR, Entwicklung einer Geometrie der allgemeinen metrischen Linienelementräume, Acta Sci. Math. Szeged, 17 (1956), pp. 85-120.

[25] B. O'Neill, Semi-Riemannian geometry, vol. 103 of Pure and Applied Mathematics, Academic Press Inc. [Harcourt Brace Jovanovich Publishers], New York, 1983. With applications to relativity.

[26] V. Perlick, Fermat principle in Finsler spacetimes, Gen. Relativity Gravitation, 38 (2006), pp. 365-380.

[27] P. Piccione, A. Portaluri, and D. V. Tausk, Spectral flow, Maslov index and bifurcation of semi-Riemannian geodesics, Ann. Global Anal. Geom., 25 (2004), pp. 121-149. 
[28] J. Szilasi, R.L. Lovas and D.Cs. Kertesz, Connections, Sprays and Finsler Structures, World scientific, 2014.

[29] J. R. Vanstone, A generalization of Finsler Geometry, Canad. J. Math., 14 (1962), pp. $87-112$.

Departamento de Matemáticas,

Universidad DE MURCIA,

Campus de Espinardo,

30100 Espinardo, Murcia, Spain

E-mail address: majava@um.es

Centro de Matemática, Computação e Cognição,

Universidade FEDERAL do ABC (UFABC),

Rua Santa AdÉlia, 166 - 09210-170 - Santo André (SP), Brazil

E-mail address: bruno.soares@ufabc.edu.br 